\title{
On application of constrained receding horizon control in astronomical adaptive optics
}

\author{
Mikhail V. Konnik ${ }^{1}$, José De Doná2 ${ }^{2}$ and James Stuart Welsh ${ }^{2}$ \\ ${ }^{1}$ School of Electrical Engineering and Computer Science, the University of Newcastle, Australia \\ ${ }^{2}$ Faculty of Engineering and Built Environment, the University of Newcastle, Australia.
}

\begin{abstract}
Control system design for adaptive optics is becoming more complex and sophisticated with increasing demands on the compensation of atmospheric turbulence. Contemporary controllers used in adaptive optics systems are optimised in the sense of a cost function (linear quadratic regulators) or to a worst case scenario (robust $H_{\infty}$ controllers). Prediction, to some extent, can be incorporated into the controllers using the Kalman filter and a model of the atmospheric turbulence.

Despite the growing number of publications on adaptive optics control systems, only the unconstrained case is usually considered. Accounting for the physical constraints of the adaptive optics system components, such as limited actuator stroke, still represents a problem. As a possible solution, one can consider constrained receding horizon control (RHC), also known as Model Predictive Control (MPC). The ability of RHC to handle constraints and make predictions of the future control signals makes it attractive for application in astronomical adaptive optics. The main potential difficulty with the application of RHC is its heavy computational load.

This paper presents preliminary results on numerical simulations of an adaptive optics system controlled by constrained RHC. In particular, the case of output disturbance rejection is considered. The results of numerical simulations are provided. Finally, methods for improving the computational performance of constrained receding horizon controllers in adaptive optics are also discussed.
\end{abstract}

Keywords: constrained control, receding horizon control, quadratic programming

\section{INTRODUCTION}

Deformable mirrors (DM) in astronomical adaptive optics systems are obviously constrained on the maximum stroke produced by the actuators. In order to account for the constraints, a receding horizon control (RHC) based on quadratic programming (QP) can be considered. In the RHC, a QP problem is solved at every sampling interval and a sequence of optimal control updates for the process is obtained. However, the main drawback of $\mathrm{RHC}$ is that the QP requires long computation times to solve the optimization problem ${ }^{1}$ at each sample point. This usually restricts the use of RHC in systems with slow dynamics and large sampling intervals.

The ever-increasing computational power provided by new technologies ${ }^{2}$ such as GPU, ${ }^{3}$ combined with the development of fast ${ }^{4}$ and efficient algorithms, ${ }^{5}$ is increasingly making RHC suitable for fast applications. ${ }^{6}$ Recently discussed interior-point ${ }^{7-9} \mathrm{QP}$ algorithms, in which the algorithm works with matrices of a fixed structure (unlike active-set methods), can be used for acceleration of the QP solving. Also it is worth considering to use reduced precision data representation: working in single precision, rather than double, gives a substantial performance improvement. ${ }^{10}$

To the best of the authors' knowledge, only a small number of papers ${ }^{11-13}$ have considered the use of constrained MPC/RHC to control deformable mirrors in adaptive optics. MPC was used ${ }^{11}$ to control a Thermal Deformable Mirror in order to correct static aberrations in a light beam. The consecutive line searches method ${ }^{14}$ in the direction of several Zernike modes was used for the constrained optimization. The Nelder-Mead ${ }^{15}$ algorithm was used in ${ }^{12}$ for correction of primary optical aberrations in a closed-loop feedback. An AO system based on the combination of a membrane DM and a stochastic parallel gradient descent control was presented in. ${ }^{13}$

Corresponding author: Mikhail V. Konnik: mail@mvkonnik.info, or mikhail.konnik@uon.edu.au Contributiong authors emails Jose.Dedona@newcastle.edu.au and James.Welsh@newcastle.edu.au

Adaptive Optics Systems III, edited by Brent L. Ellerbroek, Enrico Marchetti, Jean-Pierre Véran, Proc. of SPIE Vol. 8447, 844732 - (C) 2012 SPIE · CCC code: 0277-786/12/\$18 · doi: 10.1117/12.924955 
The purpose of this paper is to analyse the feasibility of RHC with QP for use in astronomical adaptive optics. The dynamics of the DM are considered to consist of first-order transfer functions. Several variants of coupling and constraints are simulated. The simulations presented in this paper are preliminary and do not include time-delays and measurement noise. An analysis of the structure of the resulting matrices of the RHC and the underlying QP problem is performed. A feasibility and performance study of RHC for control of deformable mirrors in adaptive optics systems is presented.

\section{MOTIVATION FOR THE USE OF CONSTRAINED RECEDING HORIZON CONTROL IN ADAPTIVE OPTICS}

It is worth asking the question: why consider constrained Receding Horizon Control (RHC), given its high computational demand? A picture is worth a thousand words: Fig. 1 shows handling the constraints on the control input, which is limited to $u \in[-100,100]$, for the same output disturbance (see Fig. 1, middle plot) by an LQR controller and an RHC controller. The LQR controller, in general, generates an abruptly changing control signal (see Fig. 1a). The RHC controller handles the constraints more efficiently (see Fig. 1b) and provides a smooth transition from the saturated states.
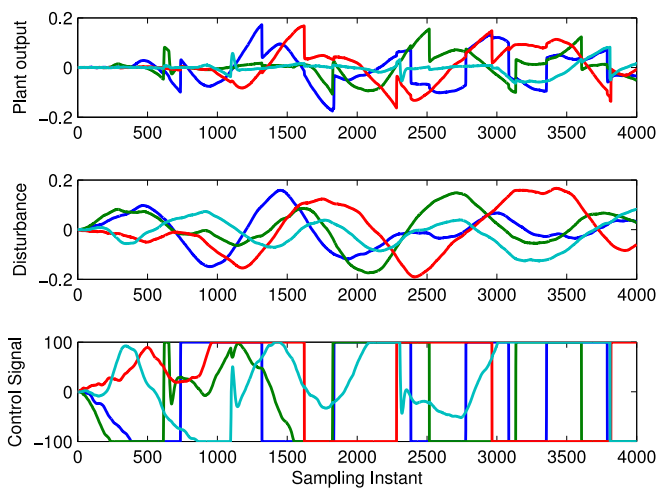

a)
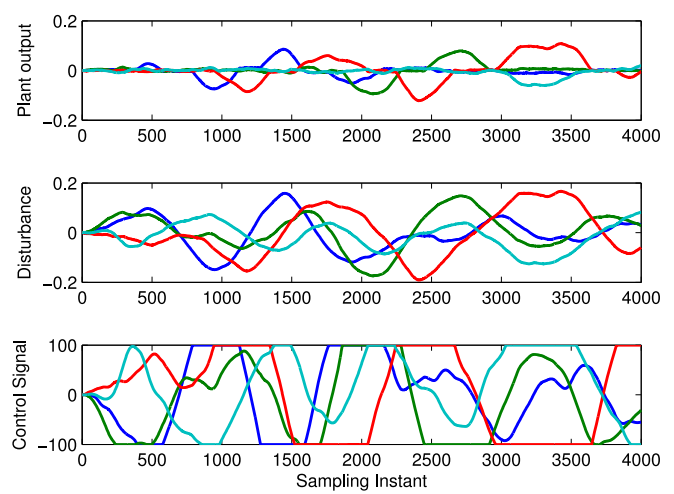

b)

Figure 1. Handling constraints on control signal for: a) an LQR controller, and b) a constrained RHC controller.

The actuators in deformable mirrors are obviously constrained by a stroke. The ability to generate control signals that are close to the maximal stroke allows utilizing the full potential of the mirror. In some cases, it may eliminate the need for the another DM in configurations like a Woofer-Tweeter. ${ }^{16,17}$

\section{FORMULATION OF RECEDING HORIZON CONTROL FOR ADAPTIVE OPTICS}

An adaptive optics system can be considered as a Linear Time-Invariant Multi-Input Multi-Output system that consists of a wavefront sensor, a controller, and a deformable mirror (DM) for the real-time compensation of an output disturbance. The goal of the controller is to track a zero reference, which is a flat wavefront.

\subsection{Dynamic models of Adaptive Optics components}

We use the model of a low-dimension weakly-coupled system. By low-dimension system we refer to a DM with up to $10 \times 10$ actuators whose dynamical interactions can be described by first-order transfer functions. The numerical model utilized in this paper does not include time-delays and measurement noise.

\subsubsection{Model of the deformable mirror dynamics}

The dynamics of the actuators in a deformable mirror (DM) are considered to be a first-order transfer functions (TF) with a fast pole. ${ }^{18}$ The dynamics of the DM actuators are usually slightly different from one actuator to the next. To account for this variation, a continuous Transfer Matrix (TM) was generated taking the values of 
TF poles from a Gaussian Distribution $p_{\mathcal{N}} \sim \mathcal{N}\left(p, \sigma_{p}^{2}\right)$ with mean $p$ and standard deviation $\sigma_{p}=\alpha \cdot p$ (here $\alpha=0.01$ is taken). This makes each $i$-th actuator's TF slightly different:

$$
G(s)_{\text {plant }}^{i}=\frac{1}{s+p_{\mathcal{N}}},
$$

where the mean value of the poles is $p=1500$.

\subsubsection{Output disturbance dynamics}

It was shown ${ }^{19}$ that an autoregressive model $\mathrm{AR}(2)$ for the output disturbance (atmospheric turbulence) provides a reasonable agreement with the model of the atmosphere. ${ }^{20}$ Therefore, the second-order continuous TF for the output disturbance that corresponds to the $i$-th actuator is:

$$
G(s)_{a t m}^{i}=\frac{1}{s^{2}+s \cdot \kappa_{1, \mathcal{N}}+\kappa_{2, \mathcal{N}}},
$$

where the values of coefficients $\kappa_{1, \mathcal{N}}$ and $\kappa_{2, \mathcal{N}}$ of the output disturbance TF were varied using a Gaussian Distributions $\kappa_{1, \mathcal{N}} \sim \mathcal{N}\left(\kappa_{1}, \sigma_{d 1}^{2}\right)$ and $\kappa_{2, \mathcal{N}} \sim \mathcal{N}\left(\kappa_{2}, \sigma_{d 2}^{2}\right)$, where $\alpha_{2}=0.01, \beta=0.1, \sigma_{d 1}=\alpha_{2} \cdot \kappa_{1}$, and $\sigma_{d 2}=\beta \cdot \kappa_{2}$. The poles of the output disturbance model are considerably slower than the poles of the DM and in this paper we used $\kappa_{1}=2$ and $\kappa_{2}=30$ as mean values of the coefficients.

\subsection{Models of coupling between actuators of the deformable mirror}

The coupling is considered static, which means that the connection between the actuators is defined as a constant coefficient, rather than dynamic (transfer function, TF). The off-diagonal continuous transfer functions, which correspond to the coupling between actuators, were constructed by application of appropriate coupling matrices $\Upsilon$ (see equations (3), (4), and (5) ) to the corresponding Transfer Matrix of the deformable mirror model. That is, for example, the coupling TF $G_{5,2}(s)$ between the actuator 5 and actuator 2 is obtained as $G_{5,2}(s)=\gamma \cdot G_{5}(s)$. Three cases of coupling between the actuators in a deformable mirror were considered, as shown in Fig. 2:

1. no coupling between the actuators, when the energized actuator has no effect on the neighbours (Fig. 2(a));

2. static coupling between nearest neighbour actuators, when the energized actuator affects the nearest neighbours (see Fig. 2(b));

3. static coupling between nearest neighbour and diagonally adjacent actuators, when the energized actuator affects both nearest neighbour actuators and diagonally adjacent actuators (see Fig. 2(c)).

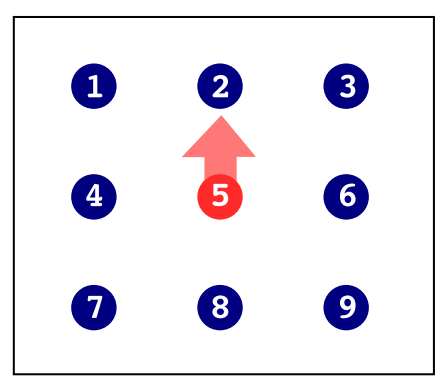

(a)

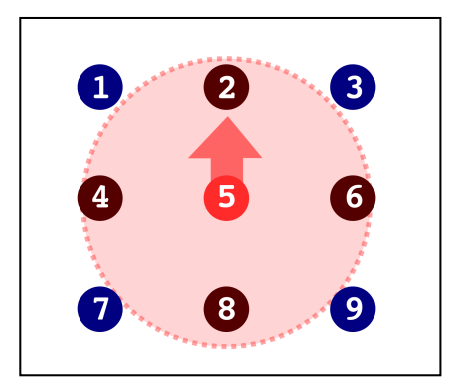

(b)

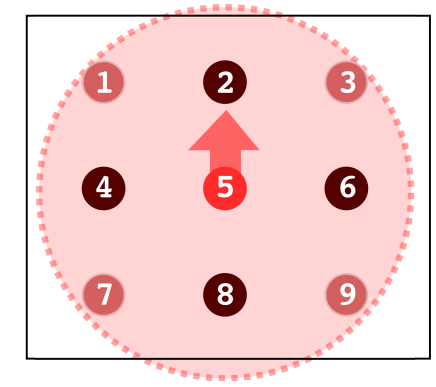

(c)

Figure 2. Coupling cases for the DM models: (a) decoupled case; (b) coupling between the nearest neighbours; (c) coupling between the nearest neighbour and diagonally adjacent actuators. 


\subsubsection{No coupling between the actuators}

When the actuators do not have an influence on each other, the Transfer Matrix (TM) in (3) contains only diagonal terms (and no coupling degrees $\gamma$ in the coupling matrix $\Upsilon_{1}$ ):

$$
\Upsilon_{1}=\left[\begin{array}{ccc}
0 & 0 & 0 \\
0 & 1 & 0 \\
0 & 0 & 0
\end{array}\right], \quad G_{\text {plant.dec }}(s)=\left[\begin{array}{cccccccc}
\mathbf{G}_{\mathbf{1}} & 0 & 0 & 0 & 0 & 0 & \ldots & 0 \\
0 & \mathbf{G}_{\mathbf{2}} & 0 & 0 & 0 & 0 & \ldots & 0 \\
\vdots & \vdots & \ddots & & & & & \vdots
\end{array}\right]
$$

Although early AO systems ignored the coupling, ${ }^{21}$ the more actuators the mirror has, the closer they are put to each other, the more strong the coupling will be in the system.

\subsubsection{Static coupling between nearest neighbour actuators}

Continuous-faceplate DMs usually have relatively strong coupling only between the nearest neighbour actuators (see Fig. 2(b)), while coupling between the diagonally adjacent actuators can be neglected. In this case the transfer matrix is denser and contains off-diagonal elements $G_{i, j}$ and the coupling degrees $\gamma$ in the matrix $\Upsilon_{2}$ :

$$
\Upsilon_{2}=\left[\begin{array}{ccc}
0 & \gamma & 0 \\
\gamma & 1 & \gamma \\
0 & \gamma & 0
\end{array}\right], \quad G_{\text {plant.nnc }}(s)=\left[\begin{array}{cccccccc}
\mathbf{G}_{\mathbf{1}} & G_{1,2} & 0 & G_{1,4} & 0 & 0 & \ldots & 0 \\
G_{2,1} & \mathbf{G}_{2} & G_{2,3} & 0 & G_{2,5} & 0 & \ldots & 0 \\
\vdots & \vdots & \ddots & & & & & \vdots
\end{array}\right]
$$

where $\gamma \in(0,1)$ is the mean value of the coupling degree between the nearest neighbour actuators.

\subsubsection{Static coupling between nearest and diagonally adjacent actuators}

When the actuators are located close to each other in a continuous-faceplate DM, the coupling between the actuators includes the diagonal adjacent neighbours as well (see Fig. 2(c)). The transfer matrix for this case is:

$$
\Upsilon_{3}=\left[\begin{array}{ccc}
\gamma_{2} & \gamma_{1} & \gamma_{2} \\
\gamma_{1} & 1 & \gamma_{1} \\
\gamma_{2} & \gamma_{1} & \gamma_{2}
\end{array}\right], \quad G_{\text {plant.nndac }}(s)=\left[\begin{array}{ccccccccc}
\mathbf{G}_{\mathbf{1}} & G_{1,2} & 0 & G_{1,4} & G_{1,5} & 0 & \ldots & 0 \\
G_{2,1} & \mathbf{G}_{\mathbf{2}} & G_{2,3} & G_{2,4} & G_{2,5} & G_{2,6} & \ldots & 0 \\
\vdots & \vdots & \ddots & & & & & \vdots
\end{array}\right]
$$

where $\gamma_{1}, \gamma_{2} \in(0,1)$ are the mean values of the coupling degree between the actuators. In this case, the coupling matrix $\Upsilon_{3}$ contains the coupling degree $\gamma_{1}$ for the nearest neighbours and $\gamma_{2}$ for the diagonally adjacent actuators.

\subsection{Overall discrete state-space model of the system dynamics}

The continuous transfer matrices (TM) of the plant, $G_{\text {plant }}(s)$, and the disturbance, $G_{a t m}(s)$, were formed using (1) and (2). Those transfer matrices were formed for the decoupled case $G_{p l a n t . d e c}(s)$ from (3), the nearest neighbour actuators coupling case $G_{\text {plant.nnc }}(s)$ from (4), and the nearest plus diagonally adjacent actuators coupling case $G_{\text {plant.nndac }}(s)$ from (5). These transfer matrices were converted to state space formulation, discretized using a zero-order hold $(\mathrm{ZOH})$ on the inputs and a sample time of $T_{s}=10^{-3}$ seconds. The overall state space model is the following:

$$
x_{k+1}=A x_{k}+B u_{k}+G \xi_{k}, \quad y_{k}=C x_{k},
$$

here $\xi_{k}$ is the process noise. The model has been augmented to account for both plant and disturbance dynamics:

$$
A=\left[\begin{array}{cc}
A_{\text {plant }} & 0 \\
0 & A_{\text {atm }}
\end{array}\right], B=\left[\begin{array}{c}
B_{\text {plant }} \\
0
\end{array}\right], G=\left[\begin{array}{c}
0 \\
B_{\text {atm }}
\end{array}\right], C=\left[\begin{array}{l}
C_{\text {plant }} C_{\text {atm }}
\end{array}\right]
$$

where matrices $A_{\text {plant }}, B_{\text {plant }}, C_{\text {plant }}$ are obtained from the discretization of the plant (deformable mirror) continuous transfer matrix $G_{\text {plant }}(s)$ (formed using equation (1)). Similarly, the matrices $A_{a t m}, B_{a t m}, C_{a t m}$ are obtained from discretization of the transfer matrix $G_{a t m}$ (formed using equation (2)) of the atmospheric turbulence that is the output disturbance. 


\subsection{Receding Horizon Control formulation for the Adaptive optics}

Using a standard state space RHC formulation, ${ }^{22}$ the cost function $V_{N_{p}, N_{c}}$ for a state prediction horizon $N_{p}$ and control prediction horizon $N_{c}$ is:

$$
V_{N_{p}, N_{c}}=\frac{1}{2} x^{T} C^{T} C x+\frac{1}{2} X^{T} \mathcal{Q} X+\frac{1}{2} U^{T} \mathcal{R} U, \quad X=\left[x_{1}, x_{2}, \ldots x_{N_{p}}\right]^{T}, \quad U=\left[u_{0}, u_{1}, \ldots u_{N_{c}}\right]^{T}
$$

where $x$ denotes the current state (i.e., $x=x_{0}$ ). The matrices $\mathcal{Q}$ and $\mathcal{R}$ are defined as:

$$
\mathcal{Q}=\operatorname{diag}\left\{C^{T} C, C^{T} C, \ldots P\right\} \text { and } \mathcal{R}=\operatorname{diag}\{R, R, \ldots R\},
$$

where the matrix $R$ is the penalty for the size of the control input, and the matrix $P$ is the penalty for missing the desired goal state (if the desired goal is zero). The penalty matrix for excessive control energy is $R=10^{-12} \cdot I$. The horizons were set to $N_{p}=2$ for the state prediction horizon and $N_{c}=1$ for the control prediction horizon.

Define matrices $\Gamma$ and $\Omega$ as:

$$
\Gamma=\left[\begin{array}{cccc}
B & 0 & \ldots & 0 \\
A B & B & \ldots & 0 \\
\vdots & \vdots & \ddots & \vdots \\
A^{N_{p}-1} B & A^{N_{p}-2} B & \ldots & A^{N_{p}-N_{c}} B
\end{array}\right] \quad \Omega=\left[\begin{array}{c}
A \\
A^{2} \\
\vdots \\
A^{N_{p}}
\end{array}\right]
$$

The dynamics in (6) can be expressed over the prediction horizon in a vector form as:

$$
X=\Gamma U+\Omega x .
$$

Substituting (11) into the cost function (8) we obtain:

$$
V_{N_{p}, N_{c}}=\bar{V}+\frac{1}{2} U^{T} \mathbb{H} U+U^{T} \mathbb{F} x,
$$

where the term $\bar{V}$ is independent of $U$. The Hessian matrix $\mathbb{H}$ and the matrix $\mathbb{F}$ are defined as follows:

$$
\mathbb{H} \triangleq \Gamma^{T} \mathcal{Q} \Gamma+\mathcal{R}, \quad \mathbb{F} \triangleq \Gamma^{T} \mathcal{Q} \Omega
$$

Unconstrained solution: the cost function in (12) is minimised when:

$$
U^{o p t}=-\mathbb{H}^{-1} \mathbb{F} x,
$$

where $x$ is typically replaced by the vector of estimated states $\hat{x}$ (usually obtained with a Kalman filter).

Constrained solution: the problem is typically formulated as a Quadratic Programming (QP) consisting of minimising the cost function (12). In this paper we consider linear constraints for the control inputs:

$$
M \cdot U \leq \eta, \text { where } M=\left[\begin{array}{c}
I_{\left(N_{c}+1\right) \cdot m} \\
-I_{\left(N_{c}+1\right) \cdot m}
\end{array}\right] \text { and } \eta=\left[\begin{array}{c}
\mathbf{u}_{\max } \\
\mathbf{u}_{\min }
\end{array}\right],
$$

where $I_{\left(N_{c}+1\right) \cdot m}$ is the $\left(N_{c}+1\right) \cdot m \times\left(N_{c}+1\right) \cdot m$ identity matrix, $N_{c}$ is the control prediction horizon, and $m$ is the number of inputs. The matrix $\mathbf{u}_{\max }=\left[u_{\max , 0}, u_{\max , 1}, \ldots u_{\max , N_{c}}\right]$ contains the maximum allowable inputs and the matrix $\mathbf{u}_{\min }=\left[-u_{\min , 0},-u_{\min , 1}, \cdots-u_{\min , N_{c}}\right]$ contains the minimum allowable inputs. The constrained solution for the RHC problem is then found by solving a QP at each sample instant of the form:

$$
\min _{U} \frac{1}{2} U^{T} \mathbb{H} U+U^{T} \mathbb{F} x, \quad \text { subject to : } M \cdot U \leq \eta
$$

where $U$ is a vector of future inputs. In the case when the Hessian matrix $\mathbb{H}$ is positive definite, which is the usually true for the adaptive optics, the quadratic optimisation problem is convex and therefore the constrained solution exists and is unique. 


\section{ANALYSIS OF THE STRUCTURE OF THE UNDERLYING QUADRATIC PROGRAMMING MATRICES}

The structure of the matrices for the constrained receding horizon control problem (more precisely, for the underlying quadratic programming problem) are analysed in this section. The structures of the $\mathbb{F}$ matrix, the Hessian matrix $\mathbb{H}$, and the inverse Hessian matrix $\mathbb{H}^{-1}$ are analysed and represented graphically. The matrices are normalised for illustrative purpose, and the zero elements are represented in white to stress the sparse structure.

\subsection{No coupling between actuators}

As one can see in Fig. 3, the Hessian and inverse Hessian are very sparse (98\% of elements are zeros). The matrix $\mathbb{F}$ contains two diagonal parts (see Fig. 3a). Since there is no coupling, the Hessian matrix $\mathbb{H}$ (see Fig. 3b) is diagonal, similar to the inverse Hessian (see Fig. 3c). In the absence of coupling the QP routine runs at maximal speed and can be efficiently paralleled.

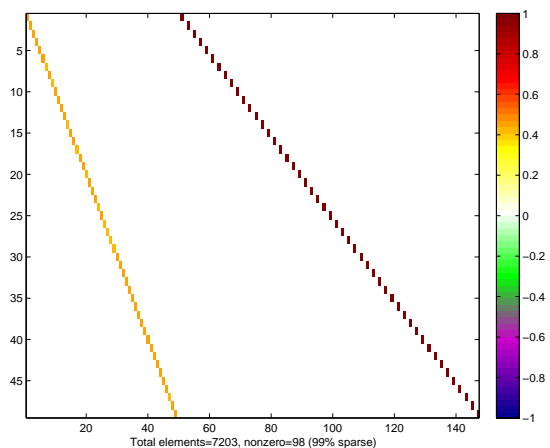

a)

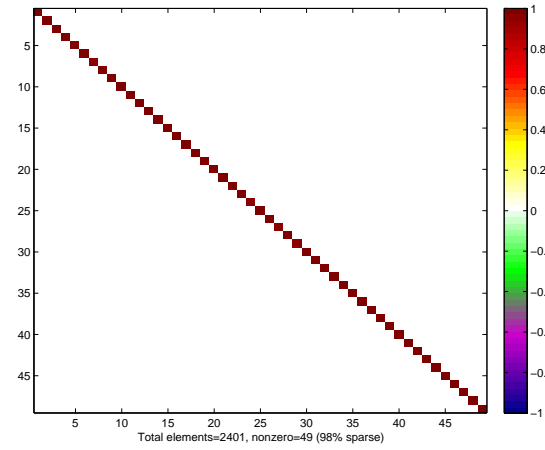

b)

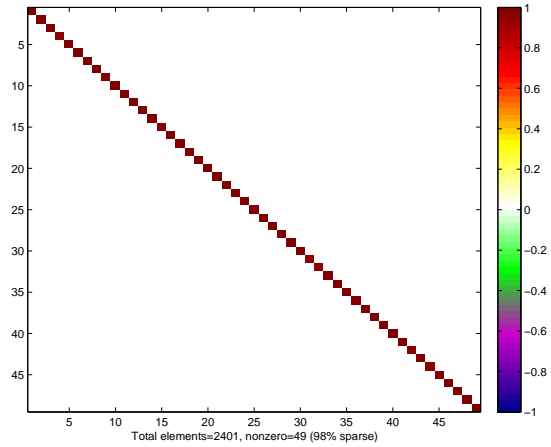

c)

Figure 3. Normalised matrices for the RHC/QP for the decoupled case of the DM with $7 \times 7$ actuators, modelled by first-order transfer functions: a) the $\mathbb{F}$ matrix, b) the Hessian matrix $\mathbb{H}$, c) the inverse Hessian matrix $\mathbb{H}^{-1}$.

\subsection{Static coupling between nearest neighbour actuators}

The coupling between the nearest actuators makes the dynamics more complex, which is reflected in the structure of the RHC matrices (see Fig. 4). The $\mathbb{F}$ matrix is very sparse with few off-diagonal elements (light yellow secondary diagonals in Fig. 4a). The analysis of the Hessian and inverse Hessian matrices presented in Fig. 5 shows that off-diagonal matrix elements can be eliminated by a threshold* to accelerate the QP problem.

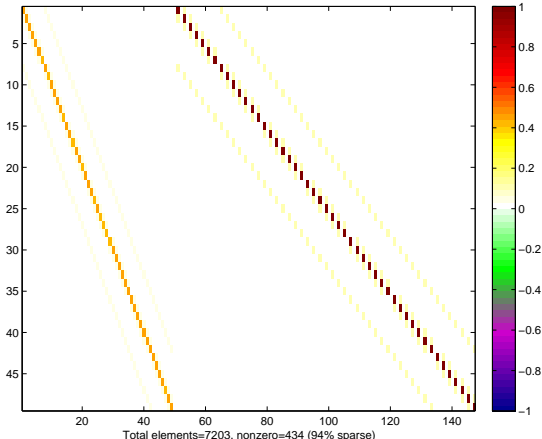

a)

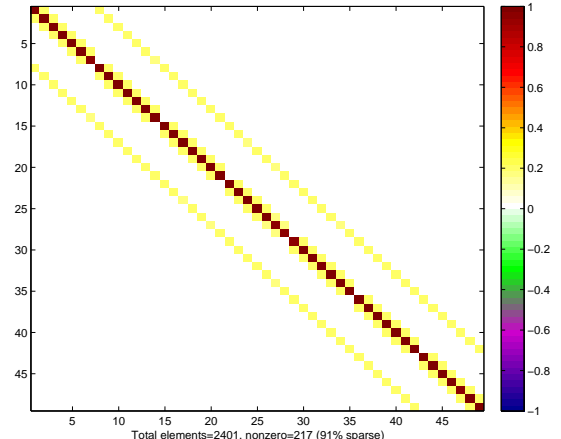

b)

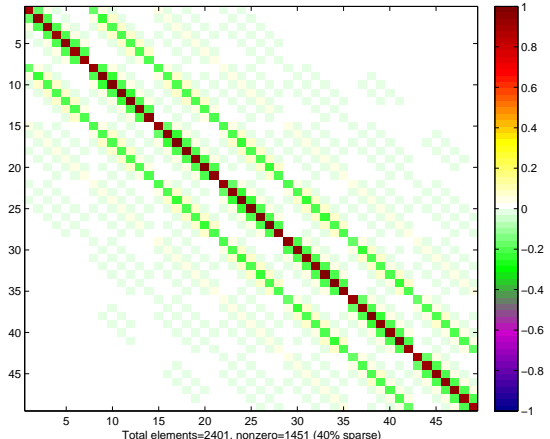

c)

Figure 4. Normalised matrices for the RHC/QP for the case when only nearest neighbour actuators of the $7 \times 7 \mathrm{DM}$ are statically coupled: a) the $\mathbb{F}$ matrix, b) the Hessian matrix $\mathbb{H}$, c) the inverse Hessian matrix $\mathbb{H}^{-1}$.

In order to find a suitable threshold, we measured the standard deviation of the residual uncompensated disturbance as a function of the threshold value applied to the Hessian matrix and the matrix $\mathbb{F}$. The more data

${ }^{*}$ The value of the threshold was calculated as $\tau=\max (\operatorname{diag}(X)) * 10^{-n}$, where $X$ is the matrix (e.g., the Hessian matrix), and $n$ is a power of ten. Then the elements of matrix $X$ that were less than the threshold were zeroed. 
are eliminated from the RHC matrices, the more sparsity can be obtained. However, excessive thresholding of matrices brings them closer to the decoupled case that leads to mismodelling (i.e., the coupled plant is controlled assuming a decoupled model). Such a mismodelling in turn causes inferior performance of the RHC controller. The goal is to find a "safe" threshold that can eliminate elements in the RHC matrices and at the same time does not reduce the disturbance rejection performance of the controller.

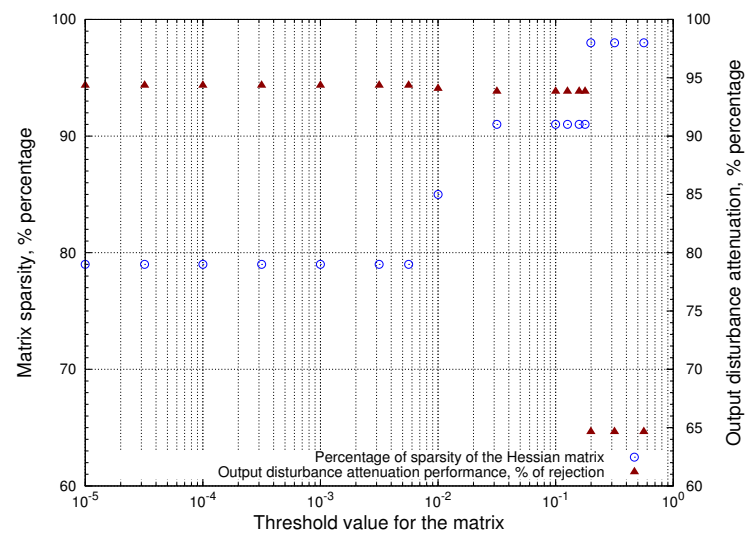

a)

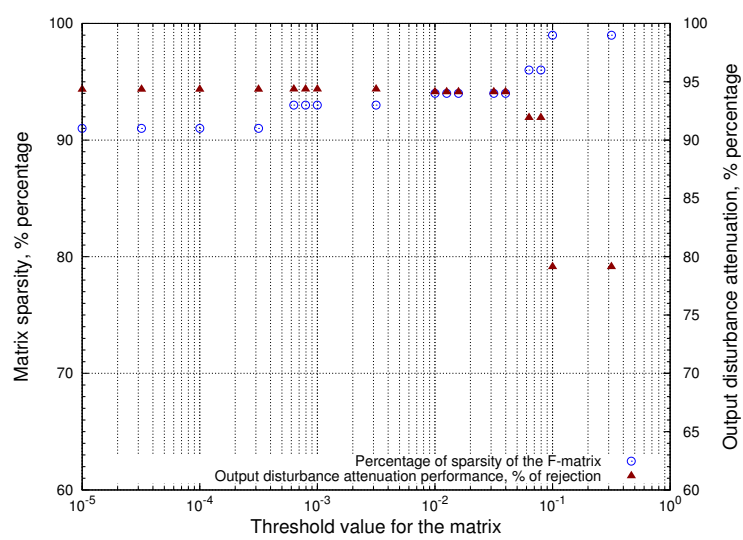

b)

Figure 5. The influence of the threshold value for the RHC matrices on sparsity and disturbance rejection performance: a) the Hessian matrix $\mathbb{H}$, b) matrix $\mathbb{F}$.

First, the "safe" threshold value was determined for the Hessian matrix. Application of a threshold up to $\tau_{\mathbb{H} \text {.safe }}=\max (\operatorname{diag}(\mathbb{H})) * 10^{-1}$ allows to increase the sparsity of the Hessian (marked by $-\circ-$ in Fig. 5a) from $79 \%$ to $92 \%$. Such a threshold does not influence the disturbance rejection performance, which is marked by $-\boldsymbol{\Delta}-$ on the right side in Fig. 5a: the output disturbance is rejected by $94 \%$. However, starting from the threshold $\tau_{\mathbb{H} \text {.unsafe }}=\max (\operatorname{diag}(\mathbb{H})) * 10^{-0.9}$, the performance of the RHC controller drops from $94 \%$ to $65 \%$. This is because we try to control coupled plant assuming a decoupled (excessively thresholded) model.

The "safe" threshold was determined for the $\mathbb{F}$ matrix in the same way as for the Hessian matrix above. One can see from Fig. $5 \mathrm{~b}$ that the threshold value $\tau_{\mathbb{F} . \text { safe }}=$ $\max (\operatorname{diag}(\mathbb{F})) * 10^{-2}$ increases sparsity (marked by - - -) of the matrix $\mathbb{F}$ from $91 \%$ to $94 \%$ while preserving the performance of the RHC controller (output disturbance rejection is $94 \%$, see Fig. 5b marked by $-\boldsymbol{\Delta}-$ ). The dependence of the sparsity, for both matrices $\mathbb{H}$ and $\mathbb{F}$, on the DM size can be seen in Fig. 6c.

The Hessian matrix is numerically well conditioned: the conditioning number grows slowly from 2.25 for the $2 \times 2$ case to about 5 for the $10 \times 10$ case. A similar behaviour is observed for the spectral radius: it grows from $3.9 \cdot 10^{-7}$ for $2 \times 2$ case to the $5.1 \cdot 10^{-7}$ for the $10 \times 10$ case. The observed numerical properties of the RHC matrices suggests that the problem is strongly convex and therefore the QP algorithms will guarantee the convergence to a unique solution.

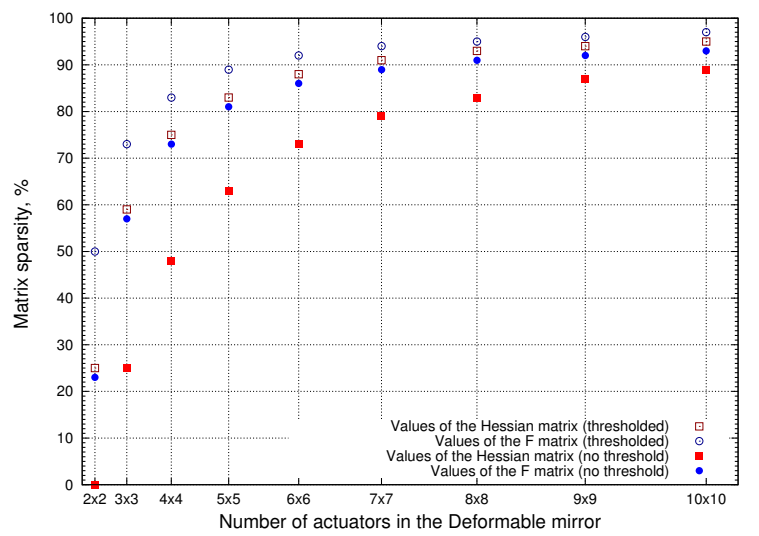

Figure 6. The influence of the threshold of RHC matrices on the attainable sparsity versus the number of actuators (coupling between nearest neighbour actuators) in a deformable mirror.

\subsection{Static coupling between nearest neighbours and diagonally adjacent actuators}

The case when the diagonally adjacent coupling is also considered. The RHC matrices are denser than for the case of only nearest neighbours, which is reflected in the sparsity patterns shown in Fig. 7 for the $7 \times 7$ DM case. Similar to the nearest neighbours coupling case in Subsection 4.2, the analysis of the off-diagonal elements and the threshold for sparsity is provided. 


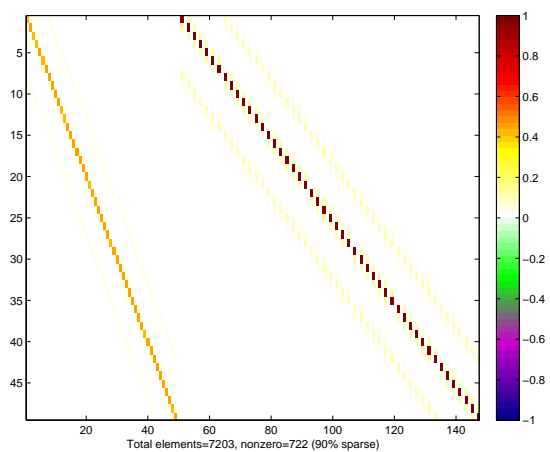

a)

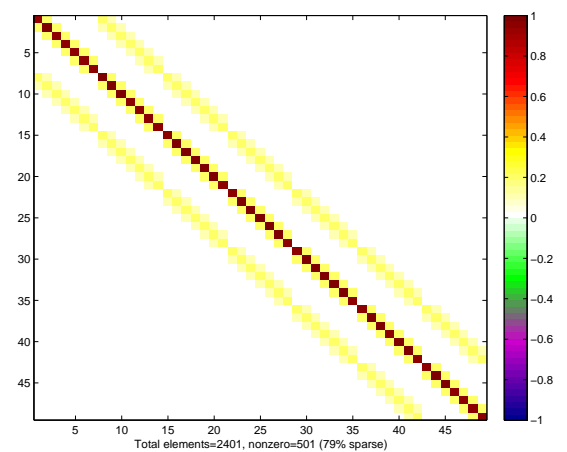

b)

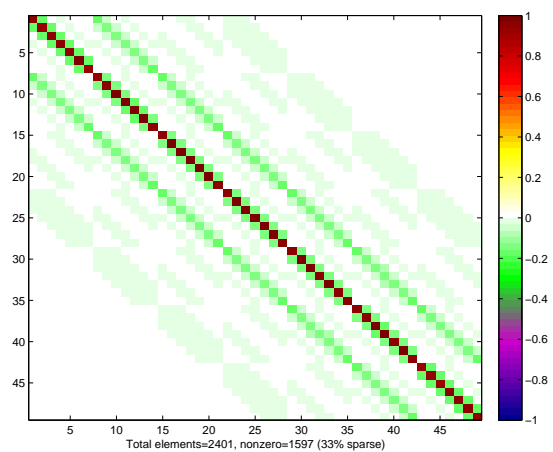

c)

Figure 7. Normalised matrices for the RHC/QP for the case when nearest and diagonally adjacent actuators are statically coupled: a) matrix $\mathbb{F}$, b) the Hessian matrix $\mathbb{H}$, c) the inverse Hessian $\mathbb{H}^{-1}$ matrix. [colour online]

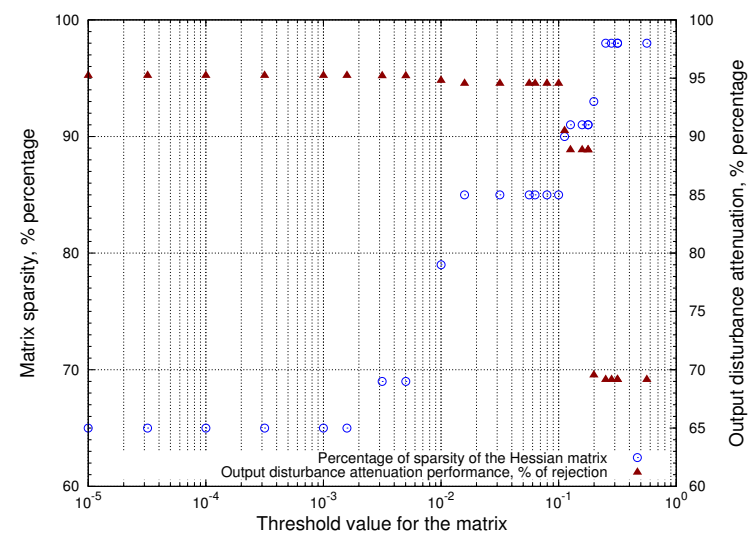

a)

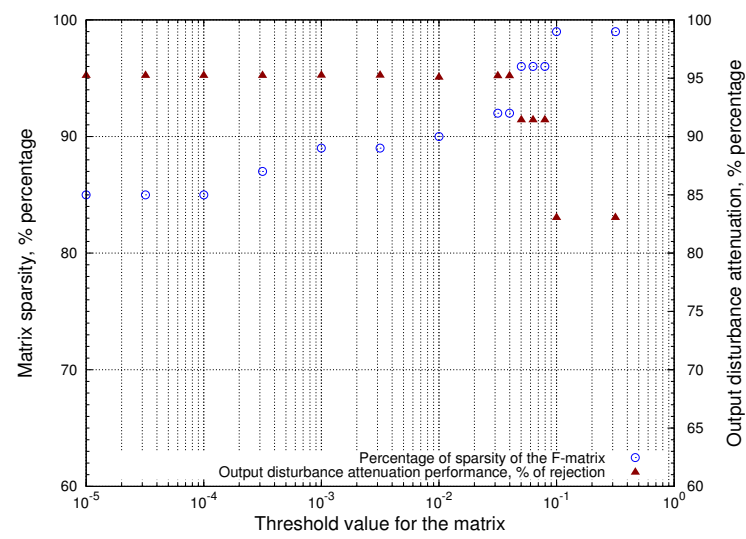

b)

Figure 8. The influence of threshold of RHC matrices on the sparsity and disturbance rejection performance for the $7 \times 7$ DM case when nearest and diagonally adjacent actuators are statically coupled: a) the Hessian matrix $\mathbb{H}, \mathbf{b})$ matrix $\mathbb{F}$.

The "safe" threshold value for the Hessian matrix was determined as $\tau_{\mathbb{H} \text {.safe }}=\max (\operatorname{diag}(\mathbb{H})) * 10^{-1.5}$, which allows increasing the sparsity of the Hessian (marked by - in Fig. 8a) from $65 \%$ to $85 \%$. Such a threshold negligibly influences the disturbance rejection performance (reduced from $95 \%$ to $94 \%$, marked by $-\boldsymbol{\Lambda}-$ on the right side in Fig. 8a). Starting from the threshold value of $\tau_{\mathbb{H} \text {.unsafe }}=\max (\operatorname{diag}(\mathbb{H})) * 10^{-1}$, the performance of the RHC controller drops from $94 \%$ to $69 \%$. As before, this happens because we try to control a coupled plant assuming a decoupled (excessively thresholded) model.

The "safe" threshold for the $\mathbb{F}$ matrix was determined as $\tau_{\mathbb{F} \text {.safe }}=\max (\operatorname{diag}(\mathbb{F})) * 10^{-1.8}$, which increases sparsity of the matrix $\mathbb{F}$ from $85 \%$ to $92 \%$ (see Fig. 8 b, marked by - $--_{-}$) while preserving the performance of the RHC controller performance. However, from the threshold value $\tau_{\mathbb{F} \text {.unsafe }}=\max (\operatorname{diag}(\mathbb{F})) * 10^{-1.5}$, elimination of the offdiagonal elements, which correspond to coupling, affects the performance of the controller: the output disturbance rejection drops from $95 \%$ to $84 \%$ (marked by - - - in Fig. 8b). The dependence of the sparsity, for both matrices $\mathbb{H}$ and $\mathbb{F}$, on the DM size can be seen in Fig. 9.

The numerical properties of the RHC matrices were analysed as well. The Hessian matrix, similarly to the 
nearest neighbours coupling case, is numerically well conditioned: the conditioning number grows from 2.1 for the $2 \times 2$ case to about 4 for the $10 \times 10$ case. The behaviour for the spectral radius is similar: it grows from $4.2 \cdot 10^{-7}$ for the $2 \times 2$ case to $6.4 \cdot 10^{-7}$ for the $10 \times 10 \mathrm{DM}$ actuators case.

\section{FEASIBILITY AND PERFORMANCE OF RECEDING HORIZON CONTROL}

The goal of the numerical simulations presented in this section is to analyse the feasibility of RHC for adaptive optics systems. Although a comparison of different QP solvers would be of interest, this has been left out from this contribution and will be reported in future works. Instead, we illustrate the feasibility of QP using the Dantzig-Wolfe algorithm from the MATLAB MPC Toolbox. Three cases of coupling where considered:

1. decoupled case: the model was described in Subsection 3.2.1, and the results are provided in Subsection 5.2;

2. coupling between nearest neighbour actuators only: the model was described in Subsection 3.2.2, and the results are provided in Subsection 5.3;

3. coupling between nearest neighbour actuators and diagonally adjacent actuators: the model was described in Subsection 3.2.3, and the results are provided in Subsection 5.4;

For each case of coupling, three additional subcases of constraints (see Fig. 10) were considered:

- weak turbulence / no constraints: the controller output never reaches the constraints (control signal limits are $U_{\lim 1}= \pm 60000$ rel.units);

- moderate turbulence / loose constraints: the limits $\left(U_{\lim 2}= \pm 230\right.$ rel.units $)$ on the control input are such that the controller output reaches the constraints $5 \%$ of the time (realistic case);

- strong turbulence / tight constraints: the limits ( $U_{\text {lim } 3}= \pm 100$ rel.units $)$ on the control input are such that the controller output reaches the constraints $20 \%$ of the time (pessimistic case).

\subsection{Simulation methodology}

\subsubsection{Hardware and software used for simulations}
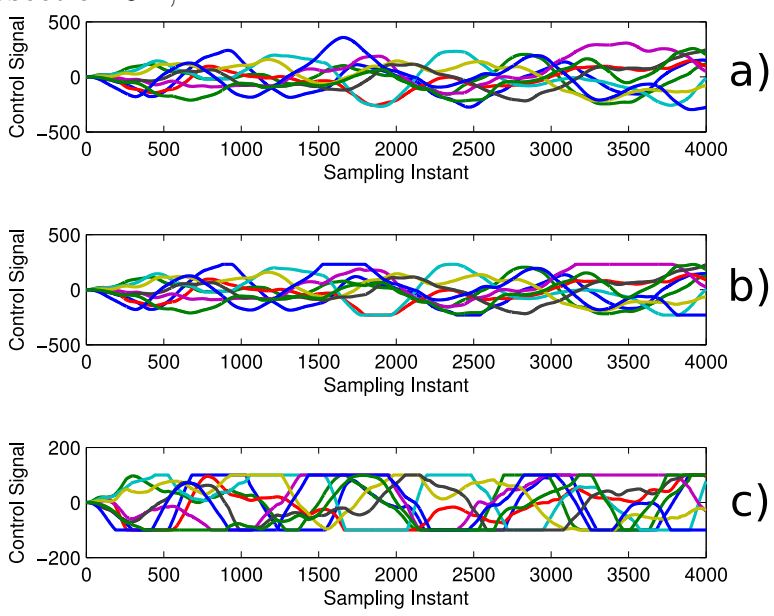

Figure 10. Constraints subcases: a) no constraints, b) loose constraints, c) tight constraints.

The MATLAB was copied into RAM using the tmpfs temporary file system in Linux to reduce the influence of the hard disc drive I/O. Using chrt, ionice, and taskset Linux commands, MATLAB was granted real-time priority and assigned to a designated core of the four-core Intel Core i7 processor ${ }^{\dagger}$. These operations reduced the uncertainty of the speed measurements considerably. The Dantzig-Wolfe active set method ${ }^{23}$ from the MATLAB 2007b Model Predictive Control Toolbox was used for the simulations.

\subsubsection{Details of the QP solver used for simulations}

The MATLAB's Dantzig-Wolfe algorithm uses the direction of the largest gradient, ${ }^{24}$ with the optimum usually found after $n+q$ iterations ${ }^{25}$ ( $n$ is the number of optimization variables and $q$ is the number of constraints). The MATLAB QP solver qpdantz.m was optimized for better performance ${ }^{\ddagger}$. The solver qpdantz.m calculates all the necessary matrices and then calls the compiled routine qpsolver.mexglx to actually solve the QP problem. The results on performance for the Dantzig-Wolfe algorithm are measured (see footnote ${ }^{\dagger}$ ) for the single-thread case (the algorithm is not parallelised) and threshold matrices ${ }^{\S}$.

${ }^{\dagger}$ The IBM/Lenovo T420 notebook was used for the numerical simulations with the following hardware inside: Intel Core i7-2640M Processor (2.80GHz, 4MB L3, 1333MHz), 8 GB DDR3 SDRAM, 500GB 7200rpm HDD, OS Debian GNU/Linux i386 v 6.0 and MATLAB 2007b for UNIX.

¥The MATLAB scripts along with the "tweaked" version of the qpdantz.m QP solver are available on the authors' development site: http://code.google.com/p/rhcaospie2012/downloads/list

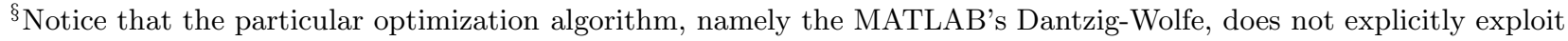
the sparsity of the matrices. Hence the use of the threshold has no noticeable impact on the speed of computations. 


\subsubsection{Simulation details}

Models of a deformable mirror with actuators from $2 \times 2$ up to $10 \times 10$ were simulated. The coupling degrees between the actuators of $\gamma_{1}=\gamma=0.20$ and $\gamma_{2}=0.1$ were considered (see Subsection 3.2 for details). The same output disturbance was used (see Subsection 3.1.2 for details) for each simulation run (i.e., the disturbance has been generated once and then used repeatedly). The number elements in the matrix, which contains data values for the output disturbance, is the same as the number of actuators (i.e., the case when the number of lenslets in a wavefront sensor is the same as the number of actuators in the DM is considered). The number of realisations of the output disturbance was 4000 for each case of the DM size (e.g., for the $2 \times 2$ case we have had 4000 matrices of size $2 \times 2$ that contained realisations of the output disturbance). The "frequencies of occurrence" vertical axis of Fig. 12, Fig. 14, and Fig. 16 means how frequently (out of 4000 realisations) the specific computational time occurs. A warm start of the controller

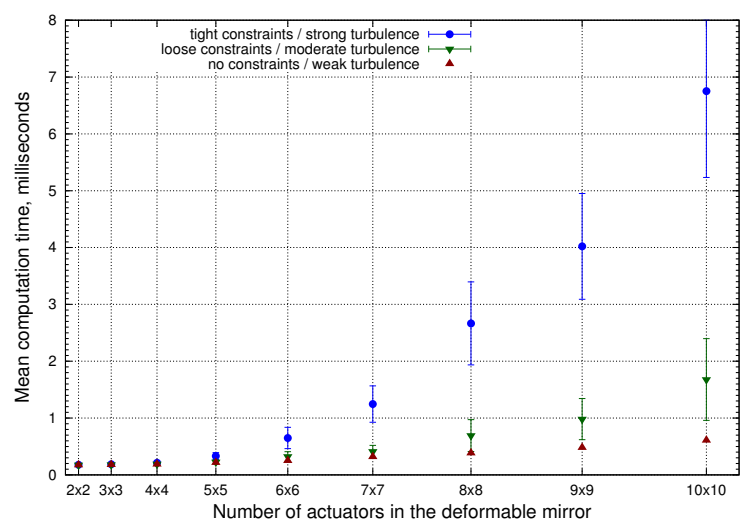

Figure 11. Decoupled case: computational time required for control inputs versus the number of actuators in the deformable mirror. is considered (i.e., the initialisation time is discarded from the discussion).

\subsection{Results of numerical simulations for the decoupled case}

The unconstrained case $(-\boldsymbol{\Delta}-)$ is the fastest, as evident from Fig. 11. The computational time grows almost linearly with the number of the actuators. Even for a relatively large case of $10 \times 10$ actuators, the computation time is below $1 \mathrm{msec}$, thus allowing the RHC controller to run at a $1 \mathrm{KHz}$.

The case of loose constraints $(-\mathbf{\nabla}-)$ requires more computational time, as seen in Fig. 11 in comparison with the unconstrained case (marked by $-\mathbf{\Delta}-$ ). From a detailed analysis in Fig. 12a (bottom part) one can see that for a small DM with 4 actuators the computational time has a very small variance. Constrained control of a deformable mirror with 49 actuators takes more time (Fig. 12a, middle part), and more important is that the required time has larger variance. This is because not all the plant inputs reach the constraints and this happens not often (on average, constraints are reached $5 \%$ of time).

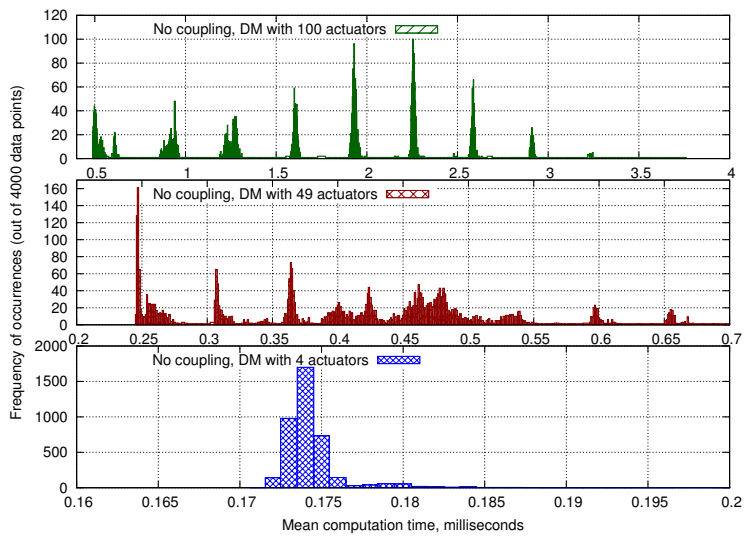

a)

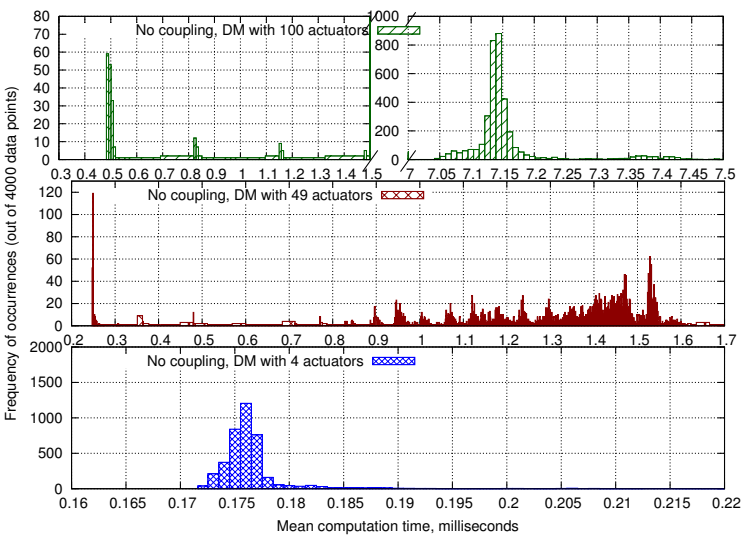

b)

Figure 12. Computation speed of the RHC with QP for the decoupled case: a) distribution of the computational time for the loose constraints case, b) distribution of the computational time for the tight constraints case.

However, other optimization algorithms, like the Hildreth-d'Esopo ${ }^{26,27}$ QP solver, can utilize the sparsity and therefore can be accelerated considerably. This issue is a subject of a current research. 
In the worst-case scenario (tight constraints, when the controller output reaches the constraints $20 \%$ of time), the computational load $-\bullet-$ is significantly larger than in the loose constraints case $-\mathbf{\nabla}-$. Since the constraints are reached by a controller very often, the computational time tends to have larger variance (see Fig. 12b). In this case, there are two most probable events: either the constraints are reached by many actuators at once (and therefore the computational time is large, see in Fig. 12b, right side of the top part), or the constraints are reached only by a few actuators (see Fig. 12b, left side of the top part). Such a variation of the computational time makes the prediction of the required time for QP solver more difficult.

\subsection{Results of numerical simulations for coupling between nearest neighbour actuators}

Similarly to the decoupled case above, the unconstrained case $\left(-\boldsymbol{\Delta}_{-}\right)$is the fastest, as seen from Fig. 13. The growth of the computational time is slow and linear with respect to the number of actuators. The maximum number of iterations for the Dantzig-Wolfe QP solver was set to 20, which was sufficient according to our simulations.

The computational burden for the loose constraints case when the constraints are reached $5 \%$ of the time is presented in Fig. 13 and marked by $-\mathbf{\nabla}-$. At least for the DantzigWolfe algorithm, the computational time grows slowly until approximately the $7 \times 7$ case but then the computational demand increases faster. One can see from the distribution of the computational time in Fig.14a (middle part) that for the case of the $7 \times 7$ actuators DM, the time has more variance (and thus the uncertainty bars $\vdash \nabla \dashv$ in Fig. 13).

The case of tight constraints, marked by $-\bullet-$, is

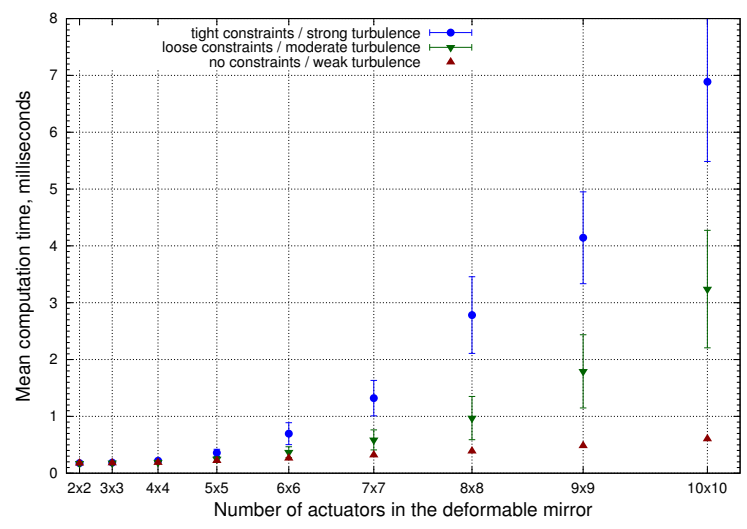

Figure 13. Nearest neighbour coupling: computational time required for control inputs versus the number of actuators in the deformable mirror.

more computationally demanding, as shown in Fig.13 and

Fig.14b. The time distribution differs from the case of loose constraints as evident from the comparison of Fig.14a and Fig.14b. The distribution of the computational time depends on how many constraints are reached: many constraints (see top right part of Fig.14b) and when few constraints are reached (see top left part of Fig.14b). Solving the QP problem for the case of tight constraints takes 2-3 times more computational time for the Dantzig-Wolfe algorithm than for the case of loose constraints as seen from the comparison in Fig.13.

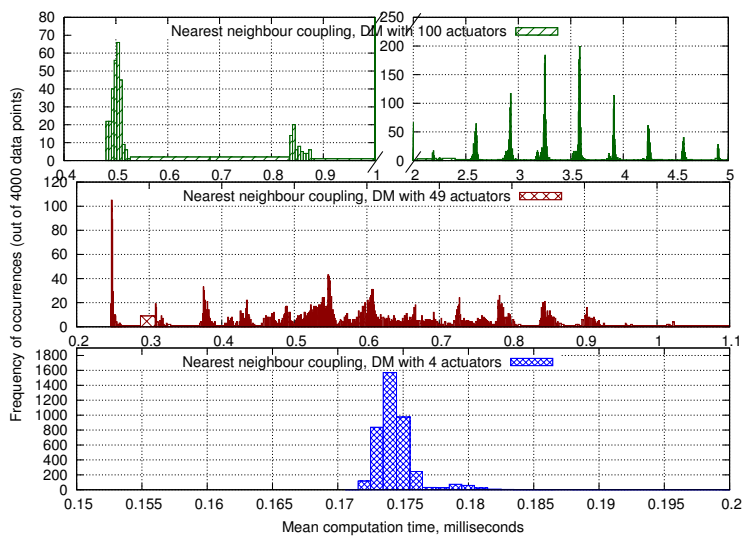

a)

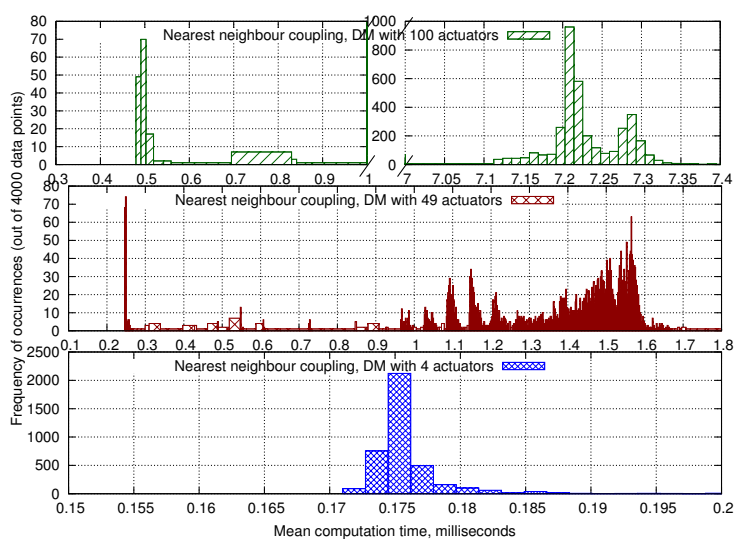

b)

Figure 14. Computation speed of the RHC/QP for coupling between nearest neighbour actuators: a) distribution of the computational time for the loose constraints case, $\mathbf{b}$ ) distribution of the computational time for the tight constraints case. 


\subsection{Results of numerical simulations for coupling between nearest neighbour and diagonally adjacent actuators}

As before, the unconstrained case $(-\boldsymbol{\Lambda}-)$ is the fastest as seen in Fig. 15. The case of loose constraints marked by $-\mathbf{\nabla}$ - is expectedly slower, but not as much as one can anticipate in comparison with the case when only nearest actuators are coupled (see Fig. 16a versus Fig. 14a).

The distribution of the computation time for the case of loose constraints has more variance due to the increased dynamics (see Fig. 16a): now one energized actuator can affect eight neighbours (four nearest and four diagonally adjacent). This increases the variance of the computational time as shown by the uncertainty bars $\vdash \nabla \dashv$ in Fig. 15 .

In the worst-case scenario of tight constraints (marked by $-\bullet-$ in Fig. 15), when the controller output reaches the constraints $20 \%$ of the time on average, the computational load is the largest with respect to the other cases. For the

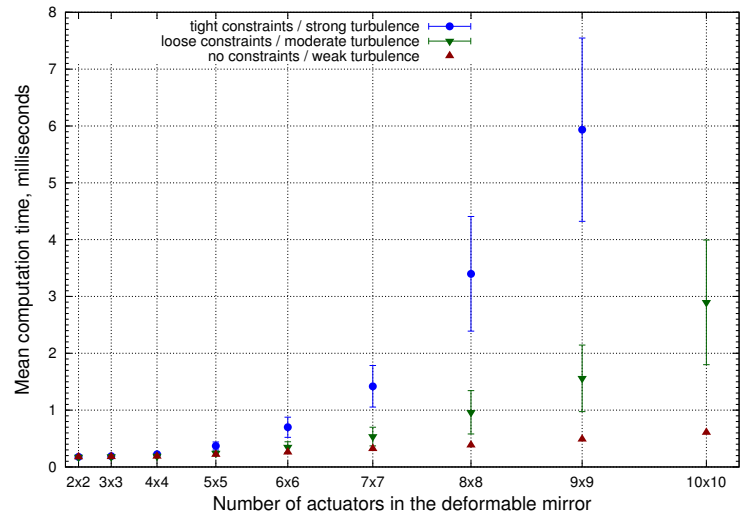

Figure 15. Nearest neighbour and diagonally adjacent coupling: computational time versus the number of actuators in the deformable mirror. case of $10 \times 10$ actuators the computational time is about $13 \mathrm{msec}$ that would be unacceptable in real-time applications. Furthermore, the maximum number of iterations for the Dantzig-Wolfe QP solver must be increased from 20 (nearest coupling case) to 35 for $7 \times 7$ actuators and 50 for the $10 \times 10$ actuators. One can also see in Fig. 16b (top part of the plot) that the computational time has a large variation (also shown by uncertainty bars $\vdash \bullet \dashv$ in Fig. 15). This makes it difficult to use the RHC controller with QP in the case of such a complicated dynamics of the DM actuators.

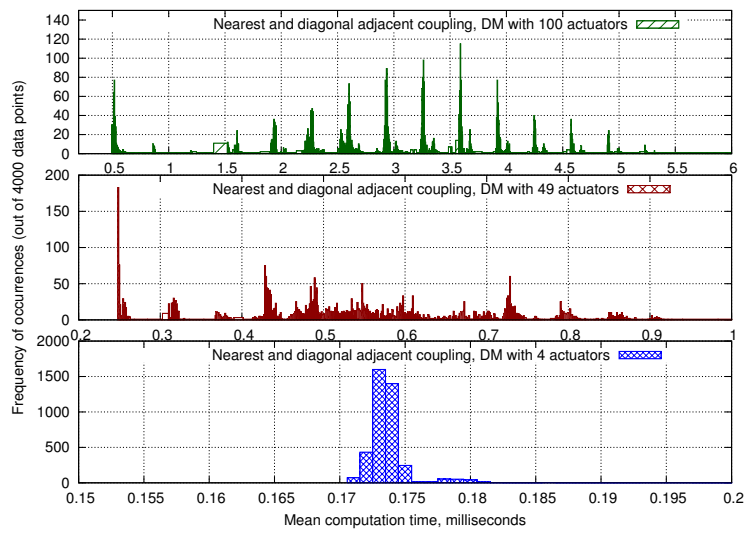

b)

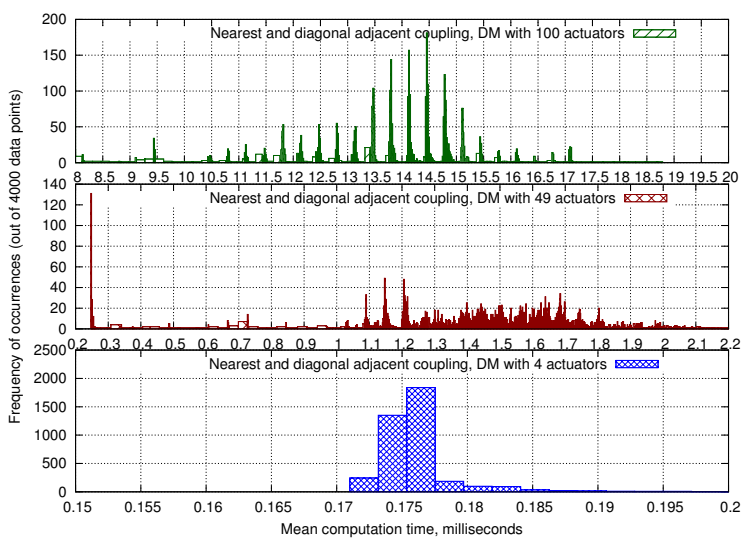

c)

Figure 16. Computation speed of the RHC with QP when coupling is between nearest neighbour and diagonally adjacent actuators: a) distribution of the computational time for the loose constraints case, b) distribution of the computational time for the tight constraints case.

\subsection{Discussion of simulations results}

The comparison of the impact of different coupling types with the same type of constraints (unconstrained case, loose and tight constraints) is provided in Fig. 17. Note that the plots have different scales on the vertical axis.

The unconstrained case is the fastest: even for the largest case of a DM with $10 \times 10$ actuators, the computational speed is below $1 \mathrm{msec}$ and grows almost linearly with the size of the problem. As evident from Fig. 17a, the speed when there are no constraints is the same for all coupling cases.

The case of loose constraints, when the limits on the control input are reached by a controller only $5 \%$ of the time, is realistic and assumes that the controller works most of time in unconstrained mode. One can see 


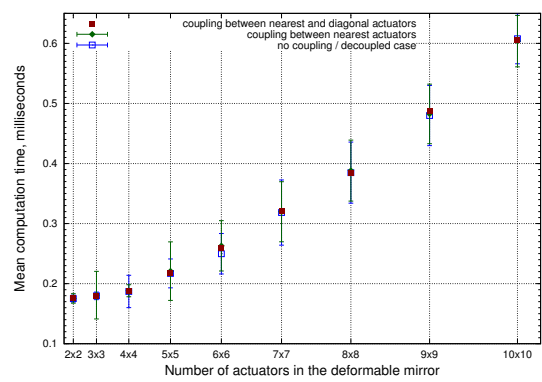

a)

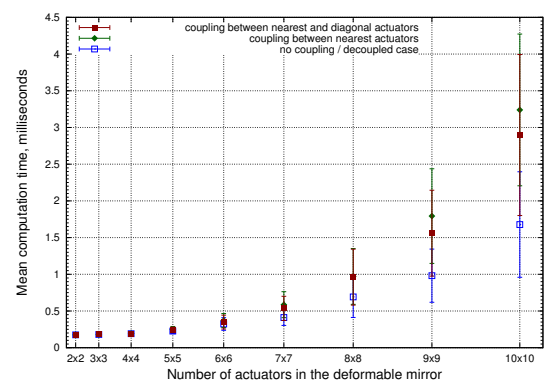

b)

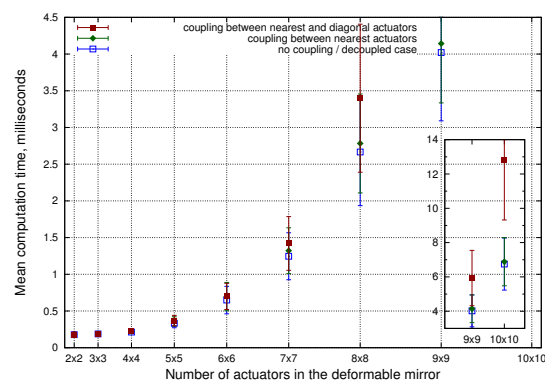

c)

Figure 17. Computation speed of the RHC with QP for different types of constraints: a) no constraints / unconstrained case, b) loose constraints / moderate turbulence, c) tight constraints / strong turbulence.

from Fig. 17b that the computation burden for the case of nearest neighbour actuators coupling marked by $-\checkmark-$ and the case of nearest and diagonally adjacent coupling marked by - - are similar for a moderate number of actuators (less than $8 \times 8$ ). The time it takes to solve the QP for such size of problems is about $1 \mathrm{msec}$ and can be decreased using more advanced algorithms, which can exploit sparsity patterns in the Hessian matrix.

The case of tight constraints is a pessimistic scenario, when the controller output reaches the constraints $20 \%$ of the time. However, one can conclude from Fig. 17c that the decoupled case $-\square-$ and the case of coupling between nearest neighbour actuators - are almost the same in terms of computational time. The case of coupling between diagonally adjacent actuators (marked by - - - in Fig. 17c) is the most difficult: one can see from Fig. 17c that the growth rate of the computational time accelerates quickly with the number of actuators.

\section{CONCLUSION}

Deformable mirrors in adaptive optics are constrained in the maximum actuators stroke, yet traditional controller do not account for these constraints. Unconstrained control inputs are usually clipped in order to prevent too large control signals and consequent mirror's damage. Such a control strategy is far from being efficient. A better solution for the constrained problem is the use of constrained Receding Horizon Control (RHC).

This paper presents preliminary results of the analysis of the matrices structure for RHC and feasibility of the underlying QP problem for control of deformable mirrors in adaptive optics. The structure of RHC matrices was analysed for the decoupled case, the case of coupling between four nearest actuators, and the case when the nearest neighbours and diagonally adjacent actuators are coupled. Using a simple threshold on matrix elements, one can attain additional sparsity (14\%-20\% more for the Hessian matrix $\mathbb{H}$ and 2-7\% more for $\mathbb{F}$ matrix) without deterioration of the performance of the controller. The matrices are numerically well-conditioned and have small spectral radius for the case of fast sampling time.

Feasibility of the QP problem was studied using a simple Dantzig-Wolfe active-set method. In the unconstrained case the computational speed is below $1 \mathrm{msec}$ (up to $10 \times 10$ actuators) and grows almost linearly with the size of the problem. In a more realistic case when the controller reaches constraints $5 \%$ of the time, the computation burden for the nearest neighbours coupling and the case of nearest and diagonally adjacent actuators is similar (the QP takes about 1 to $1.4 \mathrm{msec}$ for up to $8 \times 8$ actuators). In the worst-case scenario, when the controller reaches the constraints $20 \%$ of the time, it takes about 1 to $1.5 \mathrm{msec}$ to solve the optimisation problem for a $7 \times 7$ DM model. The computational time for QP grows quickly when the DM has more actuators.

The results presented in the paper are not the fastest achievable: there are methods for further acceleration of a QP for RHC, such as interior-point QP solvers, QP algorithms that exploit the sparsity and structure of the problem, massively parallel hardware like multi-core or GPU architectures, and reduced precision data representation. Acceleration of the QP solution for the RHC controller is the subject of current research by the authors. The results will allow designing fast and constrained RHC controllers for astronomical adaptive optics, which can utilize the full potential of the deformable mirrors. 


\section{REFERENCES}

[1] Camacho, E. and Bordons, C., [Model predictive control], Springer Verlag (2004).

[2] Kirk, D., Wen-mei, W., and Hwu, W., [Programming massively parallel processors: a hands-on approach], Morgan Kaufmann (2010).

[3] Stratton, J., Stone, S., and Hwu, W., "MCUDA: An efficient implementation of CUDA kernels for multicore CPUs," Languages and Compilers for Parallel Computing, 16-30 (2008).

[4] Ławryńczuk, M., "Computationally efficient nonlinear predictive control based on state-space neural models," Parallel Processing and Applied Mathematics , 350-359 (2010).

[5] Brand, M., Shilpiekandula, V., Yao, C., Bortoff, S., Nishiyama, T., Yoshikawa, S., and Iwasaki, T., "A parallel quadratic programming algorithm for model predictive control," in [Proc. 18th World Congress of the International Federation of Automatic Control (IFAC)], 18(1), 1031-1039 (2011).

[6] Wang, Y. and Boyd, S., "Fast model predictive control using online optimization," IEEE Transactions on Control Systems Technology 18(2), 267-278 (2010).

[7] Rao, C., Wright, S., and Rawlings, J., "Application of interior-point methods to model predictive control," Journal of optimization theory and applications 99(3), 723-757 (1998).

[8] Wright, S. J., "Applying new optimization algorithms to model predictive control," in [Proceedings of 5th Int. Conf. Chemical Process Control, CACHE, AIChE], 147 - 155 (1997).

[9] Mehrotra, S., "On the implementation of a primal-dual interior point method," SIAM Journal on optimization 2(4), 575-601 (1992).

[10] Constantinides, G., "Tutorial paper: Parallel architectures for model predictive control," in [Proceedings of the European Control Conference], 138-143 (2009).

[11] Boer, M., Hinnen, K., Verhaegen, M., Fraanje, R., Vdovin, G., and Doelman, N., "Control of a thermal deformable mirror: Correction of a static disturbance with limited sensor information," in [Adaptive Optics for Industry and Medicine], 102, 61-71 (2005).

[12] Vdovin, G. V., "Optimization-based operation of micromachined deformable mirrors," in [Proceedings of SPIE - Adaptive Optical System Technologies], 3353(1), 902-909 (1998).

[13] Plett, M., Barbier, P., and Rush, D., "Compact adaptive optical system based on blind optimization and a micromachined membrane deformable mirror," Applied Optics 40(3), 327-330 (2001).

[14] Boyd, S. P. and Vandenberghe, L., [Convex optimization], Cambridge University Press (2004).

[15] Nelder, J. A. and Mead, R., "A simplex method for function minimization," The computer journal 7(4), 308-313 (1965).

[16] Hampton, P. J., Conan, R., Bradley, C., and Agathoklis, P., "Control of a woofer tweeter system of deformable mirrors," 6274(1), $62741 \mathrm{Z}$ (2006).

[17] Lavigne, J.-F. and Véran, J.-P., "Woofer-tweeter control in an adaptive optics system using a fourier reconstructor," J. Opt. Soc. Am. A 25, 2271-2279 (Sep 2008).

[18] Hardy, J. W., [Adaptive optics for astronomical telescopes], Oxford University Press, USA (1998).

[19] Petit, C., Meimon, S., Fusco, T., Kulcsár, C., and Raynaud, H.-F., "Hybrid LQG/integrator control for the VLT eXtreme AO system SPHERE," in [IEEE International Conference on Control Applications, Yokohama, Japan, September 8-10], (2010).

[20] Frisch, U., [Turbulence: the legacy of A.N. Kolmogorov], Cambridge University Press (1995).

[21] Duffner, R. W., [The Adaptive Optics Revolution: A History], University of New Mexico Press (2009).

[22] Graham Clifford Goodwin, Maria Marta Seron, and José De Doná, [Constrained Control and Estimation: An Optimisation Approach], Springer, Berlin (2005).

[23] Fletcher, R., [Practical methods of optimization], John Wiley \& Sons, Chichester, UK (1987).

[24] Dantzig, G., [Linear programming and extensions], Princeton University Press (1963).

[25] MathWorks, MATLAB R2007b Documentation, Model Predictive Control Toolbox (2007).

[26] d'Esopo, D. A., "A convex programming procedure," Naval Research Logistics Quarterly 6(1), 33-42 (1959).

[27] Hildreth, C., "A quadratic programming procedure," Naval Research Logistics Quarterly 4(1), 79-85 (1957). 